\title{
TIME-SYNCHRONIZATION FREE LOCALIZATION IN LARGE SCALE UNDERWATER ACOUSTIC SENSOR NETWORKS
}

\author{
Wei Cheng*, Andrew Thaeler*, Xiuzhen Cheng*, Fang Liu ${ }^{\dagger}$, Xicheng $\mathrm{Lu}^{\ddagger}$ and Zexin $\mathrm{Lu}^{\ddagger}$ \\ ${ }^{*}$ Department of Computer Science \\ The George Washington University, Washington, DC, USA \\ Email: $\{$ wcheng,athaeler,cheng $\} @$ gwu.edu \\ ${ }^{\dagger}$ Department of Computer Science, University of Texas - Pan American, Edinburg, TX, USA \\ Email: fliu@cs.panam.edu \\ ${ }^{\ddagger}$ School of Computer Science, National University of Defense Technology, Changsha, Hunan, PRC \\ Email: $\{x c l u, l z x\} @ n u d t . e d u . c n$
}

\begin{abstract}
We introduce and study the localization problem in large scale underwater acoustic sensor networks. Considering that depth information is typically available for underwater sensors, we transform the $3 D$ underwater positioning problem into its two-dimensional counterpart via a projection technique. We then introduce a localization scheme specifically designed for large scale acoustic underwater sensor networks. The proposed localization scheme does not require time-synchronization in the network. This scheme relies on time-differences of arrival (TDoA) measured locally at a sensor to detect range differences from the sensor to three anchors that can mutually hear each other. We consider variations in the speed of sound and analyze the performance of the proposed scheme in terms of the number of localized nodes, location errors, and the number of reference nodes.
\end{abstract}

\section{Index Terms}

Underwater acoustic sensor networks, localization, navigation, time-synchronization free, projection, underwater positioning.

\section{Introduction}

Underwater Acoustic Sensor Networks (UWA-SNs) consist of a number of sensors and vehicles (Unmanned Underwater Vehicle (UUV), Autonomous Underwater Vehicle (AUV), etc.) which perform collaborative monitoring tasks over a given area. Relatively easy deployment, maintenance and low cost make UWA-SNs an ideal solution for a variety of applications in the complex underwater environment, such as disaster prevention, undersea exploration and assisted navigation.

Almost all underwater sensor network applications require position information. Unfortunately, location discovery of vehicles/sensors is difficult in the underwater environment. Propagation delays, motion-induced Doppler shift, phase and amplitude fluctuations, multi-path interference, etc., are present in the underwater environment and complicate location measurement. Global Positioning System (GPS) signals do not propagate well in water [1]. Some approaches for terrestrial sensor network localization include received signal strength (RSS), time of arrival (ToA), and angle of arrival (AoA). Many of these approaches are degraded in the underwater environment due to channel variations, bandwidth constraints and underwater vehicle sensor mobility [2]. For example, the Doppler shift from mobility adversely affects the AoA algorithm and distance and frequency dependent underwater signal loss make RSS-based estimation results imprecise. The accuracy of the RSS-based localization varies with the bandwidth of the signal and the signal to noise ratio at the receiver [3]. Unfortunately, the bandwidth available to most UWA-SNs is limited making acoustic positioning in UWA-SNs very challenging.

Our research is motivated by the following three observations. (i) Underwater sensors typically have depth information available through either a pressure sensor or techniques such as [1]; (ii) GIB (GPS Intelligent Buoys) [4] can be exploited as anchors on the sea surface. It is often not feasible to deploy anchor nodes at the sea floor, especially for deep ocean environments. (iii) Precise time-synchronization is difficult to achieve in underwater environments due to long propagation delays and variances in sound velocity caused by water temperature and salinity [5].

In this paper, we investigate underwater localization via projection. Our major contributions can be summarized as follows: (i) we outline a projection-based localization method that requires no time-synchronization among sensors and anchors when at least three anchors can mutually hear each other; (ii) we devise a localization scheme employing the above method that can iteratively localize large scale underwater sensor networks without the requirement for time-synchronization; (iii) we evaluate the performance of 
the proposed scheme when the speed of sound varies in sea-water.

The outline of this paper is as follows: We first summarize the major related work in Section 2. We then detail the basic positioning method in Section 3, followed by an extended localization scheme for large scale underwater networks in Section 4. Section 5 reports the performance of the proposed scheme. Our conclusions are presented in Section. 6.

\section{Related Work}

Various papers have been written on sensor location discovery in indoor and outdoor sensor networks [6], [7]. In this section, we briefly review localization techniques proposed for UWA-SNs. For a more detailed literature survey, we refer the reader to [8].

Underwater acoustic localization techniques can be broadly classified into two categories: range-based and range-free. Range based techniques measure or estimate distances or angles to a small number of anchor nodes via ToA/TDoA or even network connectivity, and then apply triangulation or multi-lateration to transform ranges into coordinates. A sufficient and necessary condition for rangebased localizability is proposed in [9], [10]. This work also proves the equivalence in localizability between distributed and centralized implementations of geometric localization methods. Range-free techniques explore the local topology and derive the position estimate from the locations of the surrounding anchor nodes. Range-based techniques generally provide better position accuracy than range-free techniques.

An area-based range-free underwater positioning (ALS) is proposed in [2]. ALS relies on anchor nodes transmitting unique signals at various power levels to partition the sensor field. A vehicle/sensor receives and decodes signals of sufficient strength. The vehicle/sensor then reports the lowest strength signals received from anchors to a central server. The central server determines which partition the node resides in based on the set of signals received.

Network connectivity can be exploited for range estimation if there is no direct communication between anchor nodes and sensors. In [11], authors propose three range detection methods based on network connectivity: DV-hop, DV-distance, and Euclidean. DV-Hop estimates distance to anchor nodes by counting the number of hops and using an average hop distance estimate. DV-distance replaces hop counts with summed distance estimates based on received signal strength. Euclidean calculates distances to anchor nodes using range estimates to neighboring nodes and the Pythagorean theorem. The paper's comparison study shows Euclidean performs better in anisotropic topologies with the tradeoff of more computation and communication. Zhou et al. [12] extend the Euclidean method to 3D UWA-SN and study its performance. This method relies on a relatively larger number of anchor nodes which increases deployment cost. Zhang and Cheng [13] propose UR-PLACE, a protocol for underwater robot self-positioning that exploits the multihop connectivity to anchor nodes via beacon flooding. The extensive local communication in [12] and the global flooding in [13] exacerbate the bandwidth shortage problem in UWA-SN, which unavoidably degrades network throughput.

Range-based underwater localization requires either longrange or short-range anchors. Since a short-range beacon covers a smaller space, a larger number of anchor nodes are required increasing deployment cost. In PARADIGM [14] vehicles interrogate anchors and measure round trip times from anchor responses to find their location for navigation. The PARADIGM network can alternatively find a vehicle by interrogating its transponder and computing its position based on the arrival times of the responses at the anchors. Motivated by terrestrial GPS, GIB [4] also measures time of arrival of a vehicle beacon at a set of anchors. GIB has the disadvantages of requiring vehicle clock synchronization with anchors and processing by a centralized server. Hahn and Rice [15] also propose a ping-pong style scheme to measure the round-trip delay for range estimation. All these long-range based methods require underwater vehicles/sensors to interrogate multiple surface buoys.

We concentrate on range-based localization in this paper to achieve better accuracy. Acoustic ranging is the preferred ranging method in underwater environments. Network timesynchronization and the speed of sound are two key requirements in acoustic ranging. Ref. [16] proposed a localization method that does not require time-synchronization, but does require an anchor deployed on the sea floor and is designed for a small network. The impact of variations in sound velocity on localization has not been addressed in research. In the next section we will present a localization method for large scale underwater networks that does not require time synchronization. This method requires three sea surface anchors and considers the variations in sound velocity.

\section{Basic Time Synchronization-Free Localiza- tion (BSFL)}

In this section, we will first briefly introduce the underwater projection technique proposed in [9], by which depth information can be employed when only three anchors are available. We then detail our design of the basic positioning method termed BSFL, which stands for Basic time Synchronization-Free Localization.

Ref. [9] proved that the to-be-localized node can preserve its localizability if the projection is non-degenerative given three non-collinear anchors. A projection is a mapping of the anchor nodes to the horizontal plane of the to-be-localized node. The projection is non-degenerative if and only if no two anchors have the same $x$ and $y$ coordinates. The task of localizing the node $S$ in a three-dimensional space can be 


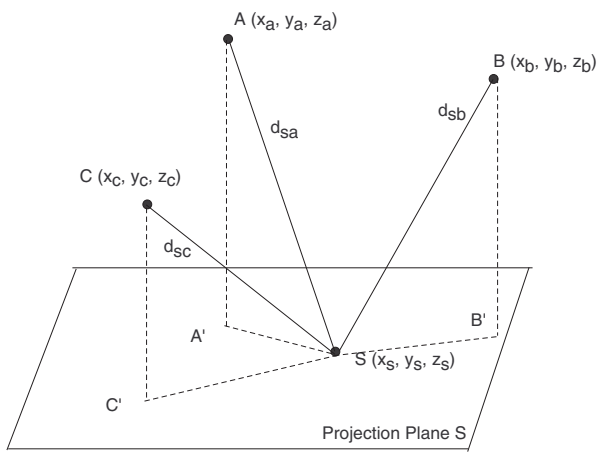

Figure 1: The sensor $\mathrm{S}$ will measure the arrival times of beacon signals from anchor nodes A, B and C. B's transmission will start after it receives A's beacon signal; C's transmission will start after it receives both A and B's beacon signals. $S$ will also receive the turn-around delay information from $\mathrm{B}$ and $\mathrm{C}$. This procedure will be repeated once every $\mathrm{T}$ seconds.

reduced to localizing the node in a two-dimensional space with a non-degenerative projection.

Fig. 1 presents an example of the projection technique. Let $A, B, C$ be three anchors at $\left(x_{a}, y_{a}, z_{a}\right),\left(x_{b}, y_{b}, z_{b}\right)$, and $\left(x_{c}, y_{c}, z_{c}\right)$, respectively, that can mutually hear each other. Let $S$ be the to-be-localized node that can hear the beacon messages from A, B, and C. If we project the three beacons onto the horizontal plane containing $S$, we will obtain three virtual anchors $A^{\prime}, B^{\prime}$, and $C^{\prime}$, which are located at position $\left(x_{a}, y_{a}, z_{s}\right),\left(x_{b}, y_{b}, z_{s}\right)$, and $\left(x_{c}, y_{c}, z_{s}\right)$, respectively. Since this projection is non-degenerative (no two of $A, B, C$ have the same $x$ and $y$ coordinates), we successfully transform the 3D underwater localization problem into a problem of localizing $S$ in a horizontal plane with virtual anchors $A^{\prime}$, $B^{\prime}$ and $C^{\prime}$. The projection distances from $S$ to the three virtual anchors can be computed by

$$
d_{s i}{ }^{\prime}=\sqrt{d_{s i}{ }^{2}-\left(z_{s}-z_{i}\right)^{2}}
$$

where $i \in\{a, b, c\}$. To apply trilateration localization to compute $\left(x_{s}, y_{s}\right)$, all the three projection distances are necessary and therefore we must compute $d_{s i}, i \in\{a, b, c\}$, according to Eq. (1).

In the following, we detail the design of BSFL. BSFL consists of two steps. The first step detects the differences in signal arrival times from three anchor nodes. These time differences are transformed into range differences from the underwater vehicle/sensor to the virtual anchor nodes. In the second step, trilateration is performed to transform these range estimates into coordinates.

Given the locations $\left(x_{a}, y_{a}, z_{a}\right),\left(x_{b}, y_{b}, z_{b}\right)$, and $\left(x_{c}, y_{c}, z_{c}\right)$ of anchor nodes $\mathrm{A}, \mathrm{B}$, and $\mathrm{C}$, respectively, we are going to determine the location $\left(x_{s}, y_{s}, z_{s}\right)$ of sensor $S$, as shown in Fig. 1, where the depth $z$ is assumed to be known. Let $d_{i j}$ be the distance between $i$ and $j$, where $i, j \in\{a, b, c, s\}$, representing the three anchor nodes and the sensor $S$. We have

$$
d_{i j}=\sqrt{\left(x_{i}-x_{j}\right)^{2}+\left(y_{i}-y_{j}\right)^{2}+\left(z_{i}-z_{j}\right)^{2}}
$$

The first step of BSFL computes the range differences between $d_{s a}, d_{s b}$, and $d_{s c}$.

\section{Step 1: Range Difference Computation.}

Let $\mathrm{A}$ be the master (golden anchor) node, which initiates a beacon signal every $T$ seconds. Each beacon interval begins when $\mathrm{A}$ transmits a beacon signal. Consider any beacon interval $i$, assume sensor $S$, anchor nodes B and C, receive A's beacon signal at times $t_{1}^{i}, t_{b}^{i}$, and $t_{c}^{i}$, respectively. At time $t_{b}^{i \prime}$, which is $\geq t_{b}^{i}$, $\mathrm{B}$ (silver anchor) replies to $\mathrm{A}$ with a beacon signal conveying information $t_{b}^{i \prime}-t_{b}^{i}=\Delta t_{b}^{i}$. This signal reaches $S$ at time $t_{2}^{i}$. After receiving beacon signals from both $\mathrm{A}$ and $\mathrm{B}$, at time $t_{c}^{i \prime}, \mathrm{C}$ (bronze anchor) replies to A with a beacon signal conveying information $t_{c}^{i \prime}-t_{c}^{i}=\Delta t_{c}^{i}$. This signal reaches $S$ at time $t_{3}^{i}$. Based on triangle inequality, $t_{1}^{i}<t_{2}^{i}<t_{3}^{i}$. Let $\Delta t_{1}^{i}=t_{2}^{i}-t_{1}^{i}, \Delta t_{2}^{i}=t_{3}^{i}-t_{1}^{i}$, we obtain

$$
\begin{aligned}
& d_{a b}+d_{s b}-d_{s a}+v \cdot \Delta t_{b}^{i}=v \cdot \Delta t_{1}^{i} \\
& d_{a c}+d_{s c}-d_{s a}+v \cdot \Delta t_{c}^{i}=v \cdot \Delta t_{2}^{i}
\end{aligned}
$$

which gives

$$
\begin{aligned}
& d_{s b}=d_{s a}+v \cdot \Delta t_{1}^{i}-d_{a b}-v \cdot \Delta t_{b}^{i}=d_{s a}+k_{1}^{i} \\
& d_{s c}=d_{s a}+v \cdot \Delta t_{2}^{i}-d_{a c}-v \cdot \Delta t_{c}^{i}=d_{s a}+k_{2}^{i}
\end{aligned}
$$

where $d_{s a}, d_{s b}$, and $d_{s c}$ are positive real numbers, $v$ is the sound velocity, which we will discuss later in this paper, and

$$
\begin{aligned}
& k_{1}^{i}=v \cdot \Delta t_{1}^{i}-v \cdot \Delta t_{b}^{i}-d_{a b} \\
& k_{2}^{i}=v \cdot \Delta t_{2}^{i}-v \cdot \Delta t_{c}^{i}-d_{a c}
\end{aligned}
$$

Averaging $k_{1}^{i}$, and $k_{2}^{i}$ over $I$ intervals gives

$$
\begin{aligned}
& k_{1}=\frac{v}{I}\left[\sum_{i=1}^{I}\left(\Delta t_{1}^{i}-\Delta t_{b}^{i}\right)\right]-d_{a b} \\
& k_{2}=\frac{v}{I}\left[\sum_{i=1}^{I}\left(\Delta t_{2}^{i}-\Delta t_{c}^{i}\right)\right]-d_{a c}
\end{aligned}
$$

In the next, we are going to apply projection and trilateration to compute the coordinates $\left(x_{s}, y_{s}\right)$ for sensor $S$. Note that, all arrival times, including $t_{j}^{i}$, where $j=1,2,3$, and $t_{j}^{i \prime}$, where $j \in\{b, c\}$, are based on the local timers of the anchor nodes and the sensor $S$. No time synchronization is required. We average multiple measurements to decrease measurement errors.

\section{Step 2: Location Computation.}

From Eqs. (5), (6), (9), and (10), we have

$$
\begin{aligned}
& d_{s b}=d_{s a}+k_{1} \\
& d_{s c}=d_{s a}+k_{2}
\end{aligned}
$$


Based on Eqs. (1), (11) and (12), we obtain three equations with three unknowns $x_{s}, y_{s}$ and $d_{s a}$.

$$
\begin{aligned}
\left(x_{s}-x_{a}\right)^{2}+\left(y_{s}-y_{a}\right)^{2} & =d_{s a}^{\prime 2} \\
\left(x_{s}-x_{b}\right)^{2}+\left(y_{s}-y_{b}\right)^{2} & =d_{s b}^{\prime 2} \\
\left(x_{s}-x_{c}\right)^{2}+\left(y_{s}-y_{c}\right)^{2} & =d_{s c}^{\prime 2}
\end{aligned}
$$

where

$$
\begin{aligned}
d_{s a} & >0 \\
d_{s a}^{\prime 2} & =d_{s a}^{2}-\left(z_{s}-z_{a}\right)^{2} \\
d_{s b}^{\prime 2} & =\left(d_{s a}+k_{1}\right)^{2}-\left(z_{s}-z_{b}\right)^{2} \\
d_{s c}^{\prime 2} & =\left(d_{s a}+k_{2}\right)^{2}-\left(z_{s}-z_{c}\right)^{2}
\end{aligned}
$$

Solving these equations we obtain

$$
\begin{aligned}
d_{s a}^{(1)} & =\frac{-\beta-\sqrt{\beta^{2}-4 \alpha \gamma}}{2 \alpha} \\
d_{s a}^{(2)} & =\frac{-\beta+\sqrt{\beta^{2}-4 \alpha \gamma}}{2 \alpha} \\
x_{s} & =M_{x} d_{s a}+N_{x} \\
y_{s} & =M_{y} d_{s a}+N_{y}
\end{aligned}
$$

where

$$
\begin{aligned}
\alpha & =M_{x}^{2}+M_{y}^{2}-1 \\
\beta & =2\left(M_{x} N_{x}^{\prime}+M_{y} N_{y}^{\prime}\right) \\
\gamma & =N_{x}^{\prime 2}+N_{y}^{\prime 2}+\left(z_{s}-z_{a}\right)^{2} \\
M_{x} & =\frac{2\left(k_{2}\left(y_{a}-y_{b}\right)-k_{1}\left(y_{a}-y_{c}\right)\right)}{M} \\
N_{x} & =\frac{\left(y_{a}-y_{b}\right) B-\left(y_{a}-y_{c}\right) A}{M} \\
M_{y} & =\frac{2\left(k_{1}\left(x_{a}-x_{c}\right)-k_{2}\left(x_{a}-x_{b}\right)\right)}{M} \\
N_{y} & =\frac{\left(x_{a}-x_{c}\right) A-\left(x_{a}-x_{b}\right) B}{M} \\
N_{x}^{\prime} & =N_{x}-x_{a} \\
N_{y}^{\prime} & =N_{y}-y_{a} \\
M & =2\left(\left(y_{a}-y_{b}\right)\left(x_{a}-x_{c}\right)-\left(y_{a}-y_{c}\right)\left(x_{a}-x_{b}\right.\right. \\
A & =k_{1}^{2}+x_{a}^{2}-x_{b}^{2}+y_{a}^{2}-y_{b}^{2}+Z_{1} \\
B & =k_{2}^{2}+x_{a}^{2}-x_{c}^{2}+y_{a}^{2}-y_{c}^{2}+Z_{2} \\
Z_{1} & =\left(z_{s}-z_{a}\right)^{2}-\left(z_{s}-z_{b}\right)^{2} \\
Z_{2} & =\left(z_{s}-z_{a}\right)^{2}-\left(z_{s}-z_{c}\right)^{2}
\end{aligned}
$$

The effectiveness of BSFL depends on the feasible space where $d_{s a}$ has a unique positive root. A sensor can be uniquely localized if it resides in the feasible space of the three anchors. Fig. 2 demonstrates the feasible space of three anchors located at $(0,0,0),(0,50,0)$, and $(50,0,0)$, respectively.

Note that BSFL is a general localization method that will work as long as the three anchors do not colinear, and can overhear each other. Also note that all the time

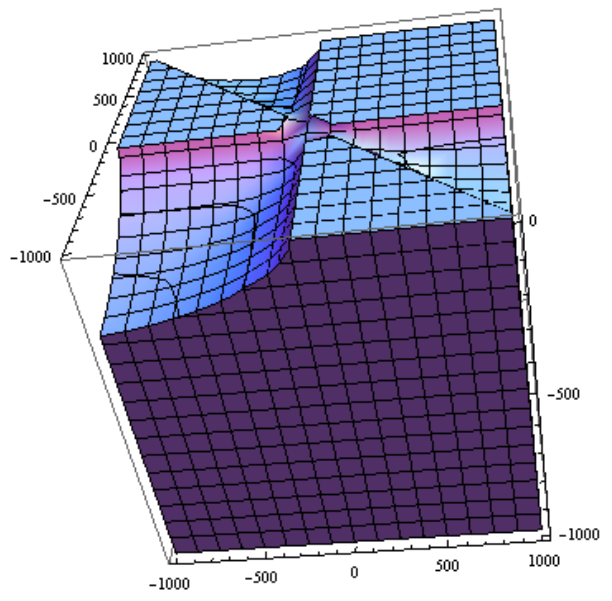

Figure 2: Feasible space for $\mathrm{S}$ where $d_{s a}$ has a unique positive root when three anchors are deployed at $(0,0,0)$, $(50,0,0)$, and $(0,50,0)$, respectively.

differences are computed from the measurements by a local timer, thus BSFL requires no time synchronization. Since BSFL does not require the underwater vehicles and sensors to transmit any beacon signal, it provides silent positioning in UWA-SNs. As discussed in [16], the silent positioning feature conserves battery life and network bandwidth, and provides location privacy to protect sensors/vehicles from being detected in critical applications.

Although BSFL provides several desirable features for 3D underwater localization, it can only be applicable to sensors that reside in the feasible space of three anchors that can mutually hear each other. Therefore BSFL does not scale well to large underwater networks. In the following section, we introduce a new scheme in which sensors with resolved location information become anchors such that more sensors can get localized iteratively.

\section{Localization Scheme for Large Scale Under- water Networks (LSLS)}

In this section, we design LSLS (Localization $\underline{\text { Scheme }}$

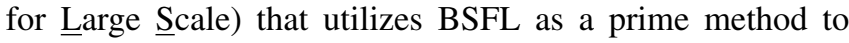
localize large scale underwater networks while retaining the nice features of BSFL such as free of time synchronization.

\subsection{LSLS Design Motivations}

The major design motivation of LSLS is to overcome the constraints of BSFL, which requires that the to-belocalized node reside in the feasible space of three anchors that can mutually hear each other. Since we target largescale underwater sensor networks, LSLS must be distributed. 
Table 1: Information recorded by each underwater node

\begin{tabular}{|c||c|}
\hline$R_{A}$ & Sea surface anchor's communications range \\
\hline$R_{S}$ & Underwater node's communications range \\
\hline$A_{\text {Center }}$ & The center of the three anchors \\
\hline Active & The node can be used as a reference node \\
\hline
\end{tabular}

A distributed implementation is necessary for a large-scale localization scheme because global information collection and distribution is costly, and central processing may not provide robustness or meet real-time requirements.

Additionally, LSLS should avoid the use of anchors on the sea floor as their deployment is difficult. Furthermore, LSLS must take into consideration the fact that underwater sensor networks are usually sparsely deployed. LSLS is an iterative procedure in which a sensor whose location is determined will become a candidate anchor in the next round. To reduce communication cost and preserve privacy, LSLS will not ask all candidate anchors to become real reference nodes; instead, it will select a small number of reference nodes to guarantee localization coverage

\subsection{LSLS}

LSLS includes three phases: sea surface anchor localization, iterative localization, and the complementary phase. In the first phase, BSFL is employed to localize the direct localizable area where nodes can be localized by the initial group of anchors. In the second phase, certain localized nodes will be selected to serve as reference nodes, and BSFL will be applied to localize the new localizable area covered by anchors and new reference nodes. The selection of new reference nodes and the localization procedure are repeated iteratively. At each round, LSLS will choose as small number of reference nodes as possible, such that most of the nodes are still passive. If a node fails to be localized in the first two phases, it can initiate a location request in the third phase. A new group of anchors will then be selected to localize the to-be-localized area of the unlocalized node. The selected groups of anchors will cover as large to-be-localized area as possible to preserve communication overhead. Note that some nodes may choose not to serve as reference nodes in LSLS due to various reasons.

Table 1 summarizes the major parameters utilized by LSLS. Assume $R_{A}$ and $R_{S}$ are both roughly known prior to the deployment and $R_{A} \geq R_{S}$. In the following, we will detail the three phases of LSLS.

\section{Sea surface anchor localization phase}

In this phase, three surface anchors in communications range send their beacon messages sequentially as described in BSFL. Nodes that receive all three beacon messages compute their locations according to Eqs. (22) and (23). A node is localized if a unique location solution is obtained. A localized node records the center of the three surface anchors

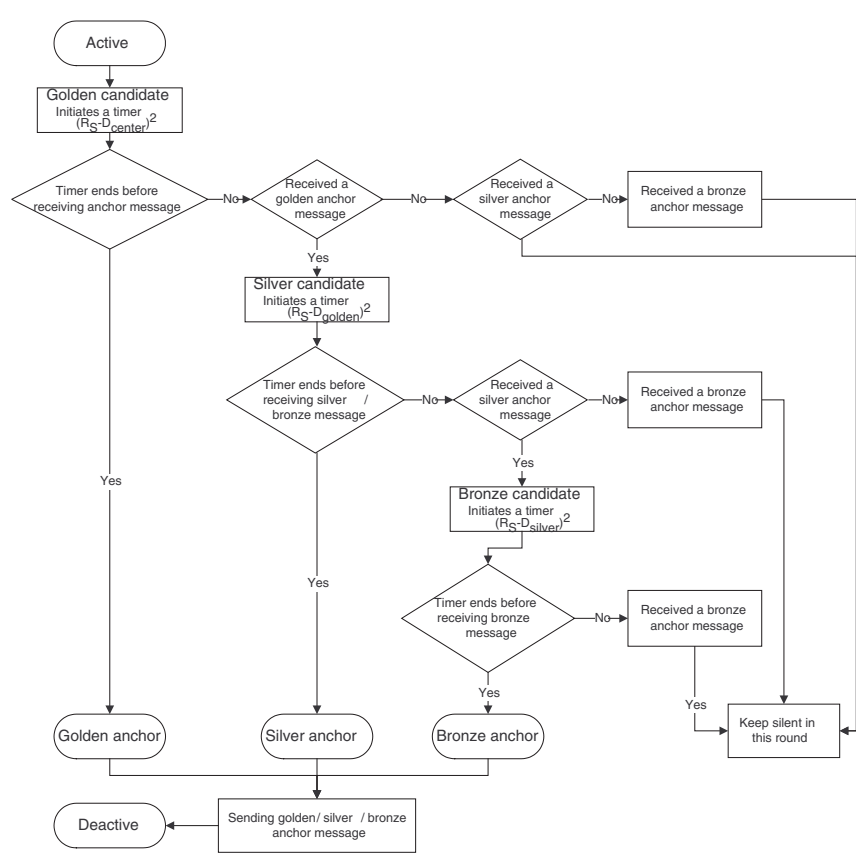

Figure 3: The behavior of an active node in the process of anchor group construction.

in $A_{\text {Center }}$, and sets itself a Active node if it intends to become a reference node and its distance to the $A_{C e n t e r}$, which is defined as $D_{\text {center }}$, is larger than $R_{A}-R_{S}$, such that the localization coverage could be increased in the next phase by adopting this node as a reference.

\section{Iterative localization phase}

In this phase, active nodes self-organize into anchor groups where three active nodes in communications range become new anchors to localize unlocalized nodes. Once a node is localized, it can become an active node and join the process of anchor group construction. Fig. 3 illustrates the behavior of an active node in the process of anchor group construction.

At the beginning of each round, every active node is a gold candidate that might work as a gold anchor in BSFL. A gold candidate initiates a timer starting from $\left(R_{S}-D_{\text {center }}\right)^{2} \mathrm{~ms}$. If it does not receive an anchor message before its timer expires, it successfully assumes the role of a golden anchor and sends its gold anchor message. This method for setting the timer helps maximize incremental coverage as the furthest active child of an anchor group will be selected such that coverage overlap with the original and new anchor group is minimized. If a gold candidate receives a gold message before its timer expires, it notes the gold anchor location and becomes a silver candidate. A silver candidate immediately initiates a timer starting from $\left(R_{S}-D_{\text {golden }}\right)^{2} \mathrm{~ms}$, where $D_{\text {golden }}$ is the distance between the silver candidate and its golden anchor. If a silver 
candidate's timer expires before receiving a silver or bronze message, it will assume the role of silver anchor and send an announcement. If a silver candidate receives a silver message before its timer expires, it becomes a bronze candidate and initiates a timer starting from $\left(R_{S}-D_{\text {silver }}\right)^{2} \mathrm{~ms}$, where $D_{\text {silver }}$ is the distance between the bronze candidate to its silver anchor. A bronze candidate assumes the role of bronze anchor if its timer expires before receiving a bronze anchor message, otherwise it remains silent for the round. A to-belocalized node localizes itself with BSFL if all three anchor message have been received. A newly localized node can become active in the following rounds.

Note that in phase II, a lower level candidate ignores higher level anchor messages, and a higher level candidate remains silent if it receives lower level anchor message in the current round. All the localized nodes can only be activated once in phase II. In a new anchor group, the silver anchor is the closest to the golden anchor, and the bronze is the closest to the silver anchor. This anchor selection strategy provides maximal coverage overlap among the three new anchors.

\section{Complementary phase}

In this phase, each of the unlocalized nodes randomly initiates a timer, and sends a localization request if no anchor message or localization request has been received before its timer ends. The active nodes that first receive a localization request are the golden anchors. The silver and bronze anchors are elected as in phase II. This anchor group construction strategy is based on the assumption that the area that contains one unlocalized node has a high probability to contain more unlocalized nodes. Then, the elected anchor group could cover most of the area that the node, which sends the localization request, can reach. All the unlocalzied nodes that are covered by the elected anchor group localize themselves by BSFL. In this phase, the unlocalized nodes that have not sent localization requests keep silence in the current round.

\section{Simulation Study}

In this section, we evaluate the performance of LSLS in terms of the number of localized nodes, location errors, and the number of active nodes. Ref. [16] stated that timebased location discovery schemes in UWA-SNs have three major sources of error: receiver system delay, underwater multipath fading, and variations in sound velocity. If anchor nodes $\mathrm{B}$ and $\mathrm{C}$ provide precise a priori information on receiver system time delays, the effect of these delays will be negligible in LSLS. We also assume that the motion of the underwater vehicles is relatively small such that the motioninduced Doppler effect can be ignored; and according to [16], LSLS can mitigate the effect of multipath fading by measuring TDoA over multiple beacon intervals because TDoA minimizes detrimental effects caused by multipath fading and processing delay [17]. Therefore, the only source of position error we consider is variations in sound velocity. Sound velocity underwater depends on temperature, salinity, pressure or depth [5]. In LSLS, we employ Coppens's model [18] to estimate the speed of sound in sea-water. Coppens's range of validity is: temperature 0 to $35 \mathrm{C}$, salinity 0 to 45 parts per thousand, depth 0 to $4000 \mathrm{~m}$. The speed of sound is modeled as:

$$
\begin{aligned}
v(D, S, t)= & v(0, S, t)+(16.23+0.253 t) D \\
& +(0.213-0.1 t) D^{2} \\
& +[0.016+0.0002(S-35)](S-35) t D
\end{aligned}
$$

where $v(0, S, t)=1449.05+45.7 t-5.21 t^{2}+0.23 t^{3}+$ $\left(1.333-0.126 t+0.009 t^{2}\right)(S-35), t=T / 10(T$ is temperature in degrees Celsius), $S$ is salinity in parts per thousand, and $D$ is depth in kilometers.

Another model for sound velocity in sea-water is the UNESCO algorithm [19], which has a more complicated form than the simple equations used in Coppens's model and uses pressure rather than depth. For the original UNESCO paper see [20]. Ref. [21] recalculated the coefficients in this algorithm following the adoption of the International Temperature Scale of 1990. If depth information is not available, LSLS can employ the guidance and suitable equations in [22] to convert pressure into depth.

\subsection{Simulation Settings}

In this study, we use Matlab to code LSLS. The following simulation parameters have been adopted:

- We generate uniformly random networks of 2000 nodes in a 3 -dimensional cubic region with a size of $100 \times$ $100 \times 200$ unit.

- We use the node communication range to control the density. The relationship between the node communication range and the network density in our simulation is shown in Fig. 4.

- There are three surface anchors that are adjacent to each other in the network. They are located at $(0,0,0)$, $(20,0,0)$, and $(0,20,0)$, respectively. The anchors' communication range is 30 .

- The reported simulation results are the average of the parameter of interest (e.g., the number of nodes localized) over 200 network instances.

\subsection{Simulation Results}

Fig. 5 reports the percentage of the total number of nodes that are localized by LSLS, where the nodes are labeled as unlocalized when their estimated locations are out of region. We observe that LSLS requires a relatively high density deployment. The major reason for this is the requirement of three reference nodes in communication range to execute BSFL. 


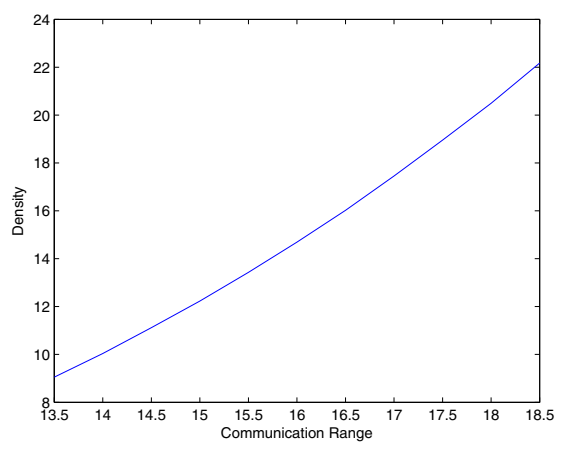

Figure 4: The relationship between node communication range and network density.

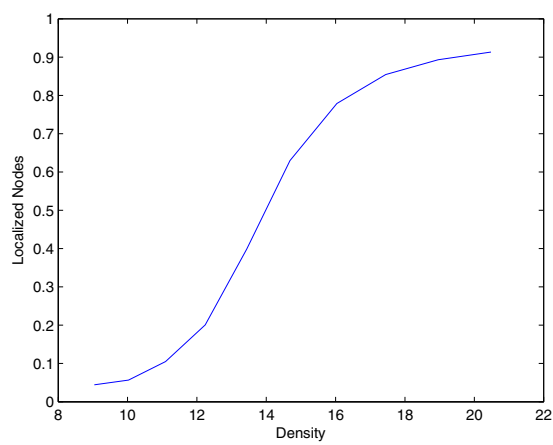

Figure 5: Number of localized nodes versus network density.

According to the analysis mentioned above, we assume the major source of error is the accuracy of underwater sound speed model. We assume the error of the speed model is equal to $N(0, \sigma) \times v$, where $v$ is the real speed of sound in underwater, and $N(0, \sigma)$ is a normal distribution function with mean 0 and variance $\sigma$. Fig. 6 illustrates the affect of $\sigma$ on the LSLS localization accuracy, where we set the network density at 17 and the localization error is unified by $\left(R_{S}\right)$. It is interesting to observe that LSLS's performance varies a lot when the accuracy of speed model is high, and does not decrease much when the speed errors are larger than 0.001. The reason for positive location error at 0 variance is that zero variance does not yield a constant zero random normal distribution variable. It means that, in our simulation, even the accuracy of the sound speed model has a 0 variance, it is still not $100 \%$ accurate due to error accumulation, an unavoidable drawback of iterative localization methods.

Since LSLS is an iterative localization method, we examine the impact of node degree on localization accuracy. Fig. 7 provides this information, where the $\sigma$ is set as 0 , and shows that at around a node of degree 16, the curve reaches its peak. The curve climbs sharply as the node degree increases

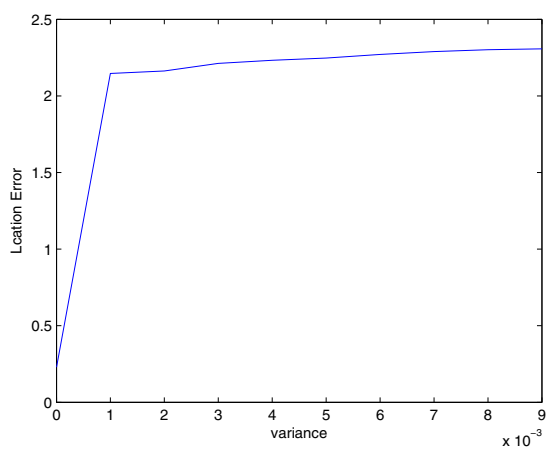

Figure 6: Location error versus speed model accuracy.

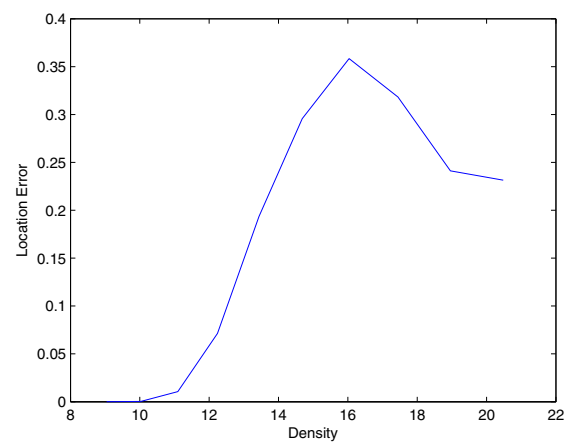

Figure 7: Location error versus network density.

from 10 to 16 , and decreases sharply from 17 to 19 , and finally decreases slowly as the node degree increases past 19. The reason for this behavior is that: At the beginning (from node degree 10 to 16 ), because the network density is relative low, only a small number of nodes can be localized by three reference nodes, thus to localize all the nodes LSLS has to keep constructing new groups of references nodes ( moreover, the increasing speed for the number of possible reference groups is low), then the number of iterations has to keep increasing and the accumulate error keeps increasing; After density 17, a relative lager number of nodes can be localized by one group of reference nodes, and the increasing of communication range makes the reference group's construction easier and reference group's localization coverage larger, then the number of iterations begins to decrease, thus the accumulate error begins to decrease.

Finally, as we have mentioned in Sec. 3, silent location discovery is desirable. Fig. 8 shows the percentage of the total number of nodes that expose their locations during the LSLS's localization process, where all nodes in the network are willing to be reference nodes. The figure shows LSLS can always provide location privacy for more than $20 \%$ nodes in the network. 


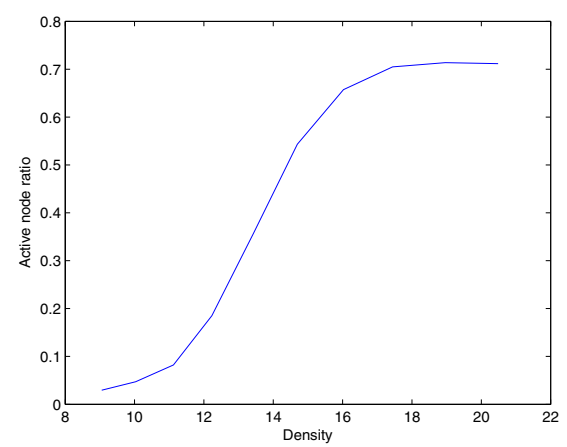

Figure 8: Number of active nodes versus network density.

\section{Summary and Future Work}

In this paper, we propose a time-synchronization free underwater localization scheme for large scale underwater networks. We analyze LSLS's performance including variations in sound velocity. In future research, we will implement our positioning scheme in a testbed and verify its performance.

\section{Acknowledgment}

The research was supported by the US National Science Foundation under grants CNS-0347674, CNS-0721669, and CNS-0831852.

\section{References}

[1] I. F. Akyildiz, D. Pompili, and T. Melodia, "Underwater acoustic sensor networks: Research challenges," Elsevier's Journal of Ad Hoc Networks.

[2] V. Chandrasekhar and W. K. Seah, "Area localization scheme for underwater sensor networks," in IEEE OCEANS Asia Pacific Conference, 2006.

[3] D. Benedetto and Giancola, "Understanding ultra wide band radio fundamentals," in Prentice Hall Communications Engineering and Emerging Technology, 2004.

[4] C. Bechaz and H. Thomas, "Gib system: The underwater gps solution," in Proceedings of 5th Europe Conference on Underwater Acoustics, 2000.

[5] "Underwater acoustics technical guides - speed of sound in seawater." [Online]. Available: http://resource.npl.co.uk/ acoustics/techguides/soundseawater/content.html

[6] X. Cheng, A. Thaeler, G. Xue, and D. Chen, "Tps: A time-based positioning scheme for outdoor wireless sensor networks," in IEEE Infocom, 2004.
[7] M. P. F. Koushanfar, S. Slijepcevic and A. SangiovanniVincentelli, "Location discovery in ad-hocwireless sensor networks," Ad Hoc Wireless Networking, 2003.

[8] V. Chandrasekhar, W. K. Seah, Y. S.Choo, and H. V. Ee, "Localization in underwater sensor networks - survey and challenges," in ACM WUWNet, 2006.

[9] W. Cheng, A. Y. Teymorian, L. Ma, X. Cheng, X. Lu, and Z. Lu, "Underwater localization in sparse $3 \mathrm{~d}$ acoustic sensor networks," in IEEE Infocom, 2008, pp. 798-806.

[10] A. Y. Teymorian, W. Cheng, L. Ma, X. Cheng, X. Lu, and Z. Lu, "3d underwater sensor network localization," 2009.

[11] D. Niculescu and B. Nath, "Ad-hoc positioning system (aps)," IEEE GlobeCom, 2001.

[12] Z. Zhou, J.-H. Cui, and S. Zhou, "Localization for largescale underwater sensor networks," in UCONN CSE Technical Report: UbiNet-TR06-04, 2006.

[13] Y. Zhang and L. Cheng, "A distributed protocol for multihop underwater robot positioning," in Proceedings of IEEE International Conference on Robotics and Biomimetics, 2004.

[14] T. C. Austin, R. P. Stokey, and K. M. Sharp, "A buoy-based system for auv navigation and tracking," in OCEANS 2000 MTS/IEEE Conference and Exhibition, 2000.

[15] M. Hahn and J. Rice, "Undersea navigation via a distributed acoustic communication network," in Proceedings of Turkish International Conference on Acoustics, 2005.

[16] X. Cheng, H. Shu, Q. Liang, and D. H.-C. Du, "Silent positioning in underwater acoustic sensor networks," in IEEE Transactions on Vehicular Technology, 2008.

[17] J. J.J. Caffery and G. L. St, "Subscriber location in cdma cellular networks," IEEE Transactions on Vehicular Technology, 1998.

[18] A. Coppens, "Simple equations for the speed of sound in neptunian waters," J. Acoust. Soc. Am, 1981.

[19] C.-T. Chen and F. Millero, "Speed of sound in seawater at high pressures," J. Acoust. Soc. Am, 1977.

[20] N. Fofonoff and R. M. Jr, "Algorithms for computation of fundamental properties of seawater," UNESCO technical papers in marine science, 1983.

[21] G. Wong and S. Zhu, "Speed of sound in seawater as a function of salinity, temperature and pressure," J. Acoust. Soc. Am, 1995.

[22] C. C. Leroy and F. Parthiot, "Depth-pressure relationship in the oceans and seas," J. Acoust. Soc. Am, 1998. 